\title{
Ankle Joint Anterior Extensor Tendons
}

National Cancer Institute

\section{Source}

National Cancer Institute. Ankle Joint Anterior Extensor Tendons. NCI Thesaurus. Code C161390.

The tendons in the anterior compartment of the leg that cross the tibiotalar joint anteriorly and connect muscles that originate on the surfaces of the tibia and fibula to bones in the toes and enable dorsiflexion of the foot at the ankle and extension of the toes. 